\title{
Fatty Acid Composition of Sunflower in 31 Inbreed and 28 Hybrid
}

\author{
Mahmmmed Harun* \\ Senior Scientific Officer, Oilseed Research Centre, Bangladesh \\ *Corresponding author: Mahmmmed Harun, Senior Scientific Officer, Oilseed Research Centre, Bangladesh
}

\begin{tabular}{|c|c|}
\hline ARTICLE INFO & ABSTRACT \\
\hline $\begin{array}{l}\text { Received: March 19, } 2019 \\
\text { Published: 幽 March 26, } 2019\end{array}$ & $\begin{array}{l}\text { High variability observed represents a very promising base to obtain new sunflower } \\
\text { inbreds with high oil quality for use either as component in hybrids or as breeding }\end{array}$ \\
\hline $\begin{array}{l}\text { Citation: Mahmmmed Harun. Fat- } \\
\text { ty Acid Composition of Sunflower in } \\
31 \text { Inbreed and } 28 \text { Hybrid. Biomed } \\
\text { J Sci \& Tech Res 16(3)-2019. BJSTR. } \\
\text { MS.ID.002851. }\end{array}$ & 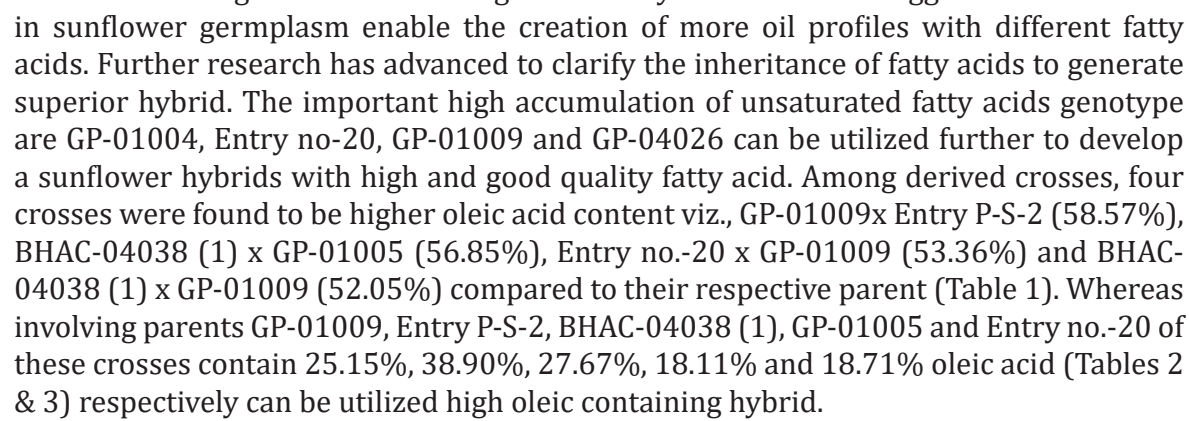 \\
\hline
\end{tabular}

\section{Introduction}

Vegetable oil consists of different types of saturated and unsaturated fatty acids (palmitic acid, stearic acid, oleic acid, linoleic acid etc.). The palmatic acid and stearic acid are the major saturated fatty acids whereas oleic and linoliec acids are unsaturated. The sunflower oil contains more of unsaturated fatty acids, mainly oleic acid and linoleic acid and very less quantity of saturated fatty acid, palmitic acid and stearic acid. Healthy oil should contain more of unsaturated fatty acids compared to saturated fatty acids. The quality of sunflower oil is generally associated with the relative concentration of oleic and linoleic acid. Oil with high oleic acid content has several benefits with regard to human health. High levels of saturated fat (Palmitic and stearic acid) consumption are correlated with increased risk of coronary heart disease. Oils with high oleic content are resistant to heat oxidation, longer shelf life and low cholesterol effect. However, Roberston reported that linoleic acid content varied inversely with the oleic acid content.

High proportion of the essential fatty acid (18:2) is considered to reduce blood cholesterol and hence sunflower has a special significance. High linoleic sunflower with $70 \%$ linoleic acid is also available in studied germplasm. It has greater oxidative stability and is useful as frying oil in the preparation of snack foods. Furthermore, sunflower oil contains fat soluble vitamins A, B, E and $\mathrm{K}$, well for heart proteins [1]. The objective of this study was to make a review of the genetic variability of oil quality components in sunflower and inheritance pattern in cross combination using own results and those of other authors.

\section{Materials and Methods}

\section{Estimation of Fatty Acid Composition}

Fatty acid profile of 31 inbreds and $28 \mathrm{~F} 1$ derived from selected eight parent through half diallel fashion was estimated by using gas chromatography. About 8-12 seeds was taken (seed was crushed in an oil paper and then transferred into a test tube). The sample was extracted and trans esterified at the same time with $5 \mathrm{ml}$ ethylated reagent (petroleum ether/0.02M sodium hydroxide in ethanol $(2 / 3)$ and shake. The samples were kept for overnight at room temperature. $10 \mathrm{ml}$ salt solution $(80 \mathrm{~g} \mathrm{NaCl}$ and $3 \mathrm{~g}$ sodium hydrogen 
sulphate in 1 liter water) was added and shake. As soon as the two layers were separated, the benzene phase was transferred to small test tubes. A Philips PU 4500 chromatograph instrument was used with Flame Ionization Detector (FID). A glass column (1.5m x 4mm) was packed with BDS. With this column the injection post, column and detector temperature was set at $220^{\circ} \mathrm{C}, 185 \mathrm{oC}$ and $240^{\circ} \mathrm{C}$, respectively. Nitrogen flow (used as career gas) rate was $22 \mathrm{ml} / \mathrm{min}$, the injection volume was $2 \mu \mathrm{l}$. Peak areas were measured with an electronic digital integrator (Shinadzu C-R6A chromatopac).

\section{Results and Discussion}

The sunflower fatty acid profile includes estimation of various proportion of palmitic acid (C16:0), stearic acid (C18:0), oleic acid (C18:1), linoleic acid (C18:2), arachidc acid (C20:0), linolenic acid (C18:3) and cis-ecosenoic (20:1) expressed in terms of percentages. The results obtained for seven fatty acids of 31 inbreds line through gas chromatography are presented in Table 4, Total fatty acid composition (Saturated and un-saturated) of the different inbred lines presented in Table 2, fatty acid composition of selected inbred parents used in half diallel crosses in sunflower are presented in Table 1 and fatty acid profile of 28 F1 derived from selected eight parent are presented in Table 4.

\section{Saturated Fatty Acid}

The hybrids with a high content of saturated fatty acids will have an important impact on the food industry because their oil will permit the production of semi-solid fats without the need of healthdetrimental processes such as hydrogenation or trans esterification [2]. But as daily intake for frying oils its percentage should be minimum. Onemli [3], Praveen [4] reported saturated fatty acid exhibit significant variation among their studied germplasm. In this study out of seven fatty acid estimated in parental inbred lines palmitic acid (C16:0), stearic acid (C18:0) and arachidic acid (C20:0) are in group of saturated fatty acid. Palmitic acid (C16:0) range $0.17-2.41$, stearic acid (C18:0) range 0.30-7.96 and arachidic acid (C20:0) range 0.02-5.52 indicates inbreds are differ from one to another for different kinds of saturated fatty acid (Table 1 ). Total saturated fatty acid range from 7.95-13.60 (Table 2). Selected inbres that used for crossing in half diallel fashion it ranges 6.27-8.46 for palmitic acid, 1.25-6.09 for stearic acid and 0.20-0.46 for arachidic acid respectively (Table 3 ). In crossing generation develop through selected inbreds it ranges 4.58-6.88 for palmitic acid, 1.96-5.17 for stearic acid and 0.20-0.39 for arachidic acid respectively.

Table 1: Fatty acid composition of the 31 inbred lines of sunflowers.

\begin{tabular}{|c|c|c|c|c|c|c|c|c|}
\hline \multicolumn{2}{|c|}{ Inbreds } & \multicolumn{7}{|c|}{ Percentage of Fatty Acids } \\
\hline \multirow[b]{2}{*}{ SI no. } & \multirow[b]{2}{*}{ Designation } & \multicolumn{3}{|c|}{ Saturated Fatty Acid (SFA) } & \multicolumn{4}{|c|}{ Unsaturated Fatty Acid (USFA) } \\
\hline & & $\begin{array}{l}\text { Palmitic Acid } \\
\text { (C16:0) }\end{array}$ & $\begin{array}{l}\text { Stearic Acid } \\
\text { (C18:0) }\end{array}$ & $\begin{array}{c}\text { Arachidc } \\
\text { Acid (C20:0) }\end{array}$ & $\begin{array}{l}\text { Oleic Acid } \\
\text { (C18:1) }\end{array}$ & $\begin{array}{l}\text { Linoleic Acid } \\
\text { (C18:2) }\end{array}$ & $\begin{array}{l}\text { Linolenic Acid } \\
\text { (C18:3) }\end{array}$ & $\begin{array}{c}\text { Cis-ecosenoic } \\
(20: 1)\end{array}$ \\
\hline G1 & BHAC-SH-037 & 6.2311 & 1.9822 & 0.32011 & 34.5679 & 55.6783 & 0.0459 & 0.0780 \\
\hline G2 & Sun-W-101 & 7.7211 & 5.4484 & 0.4336 & 31.1099 & 54.0554 & 0.1173 & 0.1446 \\
\hline G3 & Sun- W-103 & 6.0801 & 2.0269 & 0.5284 & 44.7745 & 45.2137 & 0.0852 & 0.0955 \\
\hline G4 & BHAC-04032 & 7.9893 & 1.4061 & 0.2909 & 22.2583 & 67.3469 & 0.1323 & 0.0698 \\
\hline G5 & BHAC-P-S-2 & 8.4618 & 1.9625 & 0.4207 & 25.5723 & 62.5897 & 0.0909 & 0.0264 \\
\hline G6 & BHAC-04038(1) & 6.0567 & 1.7843 & 0.4563 & 27.6745 & 63.8763 & 0.0875 & 0.0453 \\
\hline G7 & BHAC-04026 & 8.5301 & 0.8514 & 0.3384 & 28.3661 & 60.7532 & 0.1201 & 0.0584 \\
\hline G8 & BHAC-04028 & 6.0432 & 1.4194 & 0.4843 & 40.3115 & 50.5877 & 0.0594 & 0.0949 \\
\hline G9 & BHAC-04016 & 6.9573 & 3.7103 & 0.3151 & 29.0428 & 57.2838 & 0.1348 & 0.2070 \\
\hline G10 & Entry no-20 & 8.1312 & 2.3065 & 0.1999 & 18.7108 & 69.8911 & 0.1703 & 0.2201 \\
\hline G11 & Entry no-21 & 6.3434 & 4.6143 & 0.2715 & 28.2267 & 59.0254 & 0.1348 & 1.0000 \\
\hline G12 & Entry no-22 & 6.4245 & 6.2167 & 0.14689 & 37.2898 & 47.7176 & 0.4276 & 1.0000 \\
\hline G13 & Entry no-23 & 8.2963 & 1.2503 & 0.2705 & 33.1717 & 55.9953 & 0.0987 & 0.0732 \\
\hline G14 & Entry no.P-S-2 & 6.0635 & 2.5317 & 0.2669 & 38.9009 & 51.3562 & 0.0668 & 0.1246 \\
\hline G15 & GP-01002 & 7.4113 & 5.1763 & 0.5016 & 25.3153 & 60.6179 & 0.0020 & 0.1662 \\
\hline G16 & GP01004 & 6.5012 & 5.1353 & 0.341 & 40.1636 & 46.7211 & 0.0690 & 0.1100 \\
\hline G17 & GP-01005 & 7.4773 & 5.3527 & 0.3678 & 18.1084 & 67.8176 & 0.0591 & 0.0012 \\
\hline G18 & GP-01009 & 6.2744 & 6.0924 & 0.3098 & 25.1556 & 60.9614 & 0.2323 & 0.3100 \\
\hline G19 & GP-04011 & 8.6344 & 2.4451 & 0.2404 & 26.0249 & 61.9957 & 0.0914 & 0.0874 \\
\hline G20 & GP-04012 & 6.3746 & 4.8316 & 0.3228 & 29.6585 & 56.6076 & 0.0923 & 0.0891 \\
\hline G21 & GP-04015 & 6.5214 & 3.4655 & 0.2742 & 25.4531 & 63.4574 & 0.0988 & 0.1243 \\
\hline G22 & GP-04016 & 6.6776 & 3.4314 & 0.2017 & 23.4684 & 64.8983 & 0.1414 & 0.1336 \\
\hline
\end{tabular}




\begin{tabular}{|l|c|c|c|c|c|c|c|c|}
\hline G23 & GP-04017 & 6.5205 & 5.6109 & 0.2696 & 17.0873 & 67.4096 & 0.3181 & 0.2627 \\
\hline G24 & GP-04018 & 6.3144 & 4.4272 & 0.2929 & 28.4428 & 59.4558 & 0.1576 & 0.1299 \\
\hline G25 & GP-04019 & 5.7923 & 6.1755 & 0.1265 & 43.6844 & 42.3745 & 0.4234 & 0.2070 \\
\hline G26 & GP-04023 & 5.9758 & 3.8181 & 0.266 & 31.2023 & 57.1553 & 0.0894 & 0.1048 \\
\hline G27 & GP-04024 & 6.8573 & 3.9103 & 0.4151 & 28.0428 & 55.2838 & 0.1447 & 0.2080 \\
\hline G28 & GP-04026 & 7.3145 & 5.0245 & 0.3024 & 19.5954 & 66.2245 & 0.1585 & 0.7934 \\
\hline G29 & GP-04028 & 5.2543 & 3.7103 & 0.3798 & 24.6706 & 60.8798 & 0.1480 & 0.1227 \\
\hline G30 & GP-04030 & 6.4347 & 5.4827 & 0.2564 & 24.2609 & 62.2216 & 0.0954 & 0.0004 \\
\hline G31 & GP-04038(2) & 7.9266 & 3.7103 & 0.2618 & 29.0428 & 18.3698 & 0.0988 & 0.1545 \\
\hline \multicolumn{2}{r|}{ Over all mean } & 6.95 & 3.72 & 0.38 & 29.01 & 57.22 & 0.13 & 0.21 \\
\hline & SD & 0.1675 & 0.2960 & 0.0176 & 1.3032 & 1.7989 & 0.0173 & 0.0492 \\
\hline \multicolumn{2}{r|}{ CV(\%) } & 2.41 & 7.96 & 5.52 & 4.49 & 3.14 & 12.79 & 23.73 \\
\hline \multicolumn{2}{r|}{ Maximum } & 8.63 & 6.22 & 0.53 & 44.77 & 69.89 & 0.43 & 1.00 \\
\hline \multicolumn{2}{r|}{ Minimum } & 5.25 & 0.85 & 0.13 & 17.08 & 18.37 & 0.002 & 0.0004 \\
\hline
\end{tabular}

Table 2: Total fatty acid composition (Saturated and un-saturated) of the different inbred lines.

\begin{tabular}{|c|c|c|c|c|}
\hline \multicolumn{2}{|r|}{ Inbreds } & \multicolumn{3}{|c|}{ Percentage of Fatty Acids } \\
\hline SI no. & Designation & Total saturated fatty acid (SFA) & Total un-saturated fatty Acid (SFA) & Total fatty acid (TFA) \\
\hline G1 & BHAC-SH-037 & 8.5334 & 90.3701 & 98.9035 \\
\hline G2 & Sun-W-101 & 13.6031 & 85.4272 & 99.0303 \\
\hline G3 & Sun- W-103 & 8.6354 & 90.1689 & 98.8043 \\
\hline G4 & BHAC-04032 & 9.6863 & 89.8073 & 99.4936 \\
\hline G5 & BHAC-P-S-2 & 10.845 & 88.2793 & 99.1243 \\
\hline G6 & BHAC-04038(1) & 8.2973 & 91.6836 & 99.9809 \\
\hline G7 & BHAC-04026 & 9.7199 & 89.2978 & 99.0177 \\
\hline G8 & BHAC-04028 & 7.9469 & 91.0535 & 99.0004 \\
\hline G9 & BHAC-04016 & 10.9827 & 86.6684 & 97.6511 \\
\hline G10 & Entry no-20 & 10.6376 & 88.9923 & 99.6299 \\
\hline G11 & Entry no-21 & 11.2292 & 88.4596 & 99.6888 \\
\hline G12 & Entry no-22 & 12.7880 & 86.545 & 99.33309 \\
\hline G13 & Entry no-23 & 9.8171 & 89.3389 & 99.156 \\
\hline G14 & Entry no.P-S-2(1) & 8.8621 & 90.4485 & 99.3106 \\
\hline G15 & GP-01002 & 13.0892 & 86.1014 & 99.1906 \\
\hline G16 & GP01004 & 11.9775 & 87.0637 & 99.0412 \\
\hline G17 & GP-01005 & 13.1978 & 85.9863 & 99.1841 \\
\hline G18 & GP-01009 & 12.6766 & 86.6593 & 99.3359 \\
\hline G19 & GP-04011 & 11.3199 & 88.1994 & 99.5193 \\
\hline G20 & GP-04012 & 11.529 & 86.4475 & 97.9765 \\
\hline G21 & GP-04015 & 10.2611 & 89.1336 & 99.3947 \\
\hline G22 & GP-04016 & 10.3107 & 88.6417 & 98.9524 \\
\hline G23 & GP-04017 & 12.401 & 85.0777 & 97.4787 \\
\hline G24 & GP-04018 & 11.0345 & 88.1861 & 99.2206 \\
\hline G25 & GP-04019 & 12.0943 & 86.6893 & 98.7836 \\
\hline G26 & GP-04023 & 10.0599 & 88.5518 & 98.6117 \\
\hline G27 & GP-04024 & 11.1827 & 83.6793 & 94.862 \\
\hline G28 & GP-04026 & 12.6414 & 86.7718 & 99.4132 \\
\hline G29 & GP-04028 & 9.3444 & 85.8211 & 95.1655 \\
\hline G30 & GP-04030 & 12.1738 & 86.5783 & 98.7521 \\
\hline G31 & GP-04038(2) & 11.8987 & 47.6659 & 59.5646 \\
\hline
\end{tabular}




\begin{tabular}{|c|c|c|c|}
\hline Over all mean & 10.93 & 86.57 & 97.50 \\
\hline Standard Deviation & 1.57 & 7.47 & 7.13 \\
\hline CV & 14.34 & 8.63 & 7.31 \\
\hline Minimum & 7.95 & 47.67 & 59.56 \\
\hline Maximum & 13.60 & 91.68 & 99.98 \\
\hline
\end{tabular}

Table 3: Fatty acid composition of selected inbred parents used in half diallel crosses in sunflower.

\begin{tabular}{|c|c|c|c|c|c|c|c|c|}
\hline \multicolumn{2}{|r|}{ Inbreds } & \multicolumn{7}{|c|}{ Percentage of Fatty Acids } \\
\hline \multirow{2}{*}{$\begin{array}{r}\text { Sl } \\
\text { no. }\end{array}$} & \multirow[b]{2}{*}{ Designation } & \multicolumn{3}{|c|}{ Saturated Fatty Acid (SFA) } & \multicolumn{4}{|c|}{ Unsaturated Fatty Acid (USFA) } \\
\hline & & $\begin{array}{l}\text { Palmitic Acid } \\
\text { (C16:0) }\end{array}$ & $\begin{array}{l}\text { Stearic Acid } \\
\text { (C18:0) }\end{array}$ & $\begin{array}{c}\text { Arachidc } \\
\text { Acid (C20:0) }\end{array}$ & $\begin{array}{l}\text { Oleic Acid } \\
\text { (C18:1) }\end{array}$ & $\begin{array}{l}\text { Linoleic Acid } \\
\text { (C18:2) }\end{array}$ & $\begin{array}{c}\text { Linolenic } \\
\text { Acid (C18:3) }\end{array}$ & $\begin{array}{c}\text { Cis-ecosenoic } \\
(20: 1)\end{array}$ \\
\hline G1 & BHAC-P-S-2 & 8.4618 & 1.9625 & 0.4207 & 25.5723 & 62.5897 & 0.0909 & 0.0264 \\
\hline G2 & BHAC-04038(1) & 6.0567 & 1.7843 & 0.4563 & 27.6745 & 63.8763 & 0.0875 & 0.0453 \\
\hline G3 & Entry no-20 & 8.1312 & 2.3065 & 0.1999 & 18.7108 & 69.8911 & 0.1703 & 0.2201 \\
\hline G4 & Entry no-23 & 8.2963 & 1.2503 & 0.2705 & 33.1717 & 55.9953 & 0.0987 & 0.0732 \\
\hline G5 & GP-01004 & 6.5012 & 5.1353 & 0.341 & 40.1636 & 46.7211 & 0.069 & 0.1100 \\
\hline G6 & GP-01005 & 7.4773 & 5.3527 & 0.3678 & 18.1084 & 67.8176 & 0.0591 & 0.0012 \\
\hline G7 & GP-01009 & 6.2744 & 6.0924 & 0.3098 & 25.1556 & 60.9614 & 0.2323 & 0.3100 \\
\hline G8 & GP-04026 & 7.3145 & 5.0245 & 0.3024 & 19.5954 & 66.2245 & 0.1585 & 0.7934 \\
\hline \multicolumn{2}{|r|}{ Over all mean } & 7.56 & 3.61 & 0.33 & 26.02 & 61.76 & 0.12 & 0.20 \\
\hline \multicolumn{2}{|r|}{ SD } & 0.83 & 1.96 & 0.08 & 7.67 & 7.44 & 0.06 & 0.26 \\
\hline \multicolumn{2}{|r|}{$\mathrm{CV}(\%)$} & 10.91 & 54.19 & 24.63 & 29.49 & 12.05 & 49.76 & 132.95 \\
\hline \multicolumn{2}{|r|}{ Minimum } & 6.27 & 1.25 & 0.20 & 18.11 & 46.72 & 0.06 & 0.001 \\
\hline \multicolumn{2}{|r|}{ Maximum } & 8.46 & 6.09 & 0.46 & 40.16 & 69.89 & 0.23 & 0.79 \\
\hline
\end{tabular}

\section{Palmitic Acid}

Standard sunflower genotypes contains to 5-6\% palmitic acid. Onemli [3], Praveen [4] observed significant variation for palmitic acid (C16:0) in sunflower genotype. A low level palmitic acid is preferred from human health point of view. Palmitic acid is believed to increase LDL (low density lipoprotein), which is associated with cardiovascular disease risk. In the present study the palmitic acid ranges in parental line 5.25-8.63\%. Proportion of palmitic acid was low in parental line lines GP-04028 (5.25\%) and GP-04019 (5.79\%), in selected parent that used for crossing in half diallel fashion lines the lowest recorded was GP-01009 (6.27\%) and GP-04026 (7.31\%), while in 28 derived crosses, it ranged from 4.58 to 6.88 percent. The lowest proportion of palmitic acid in derived crosses was BHAC04038(1)xGP-01005 (4.58\%), Entry no-20xGP-01004 (4.67\%) and GP-01009xEntry-P-S-2 (4.97\%). Praveen (2015) reported palmitic acid range 4.43-10.93\%, Skoric, mentioned palmitic acid may vary 3.0-11.5\% and according to Anon. 2015-16 palmitic acid ranges 5-8 percent in sunflower.

\section{Stearic Acid}

Stearic acid is categorized as saturated fatty acid, the higher concentrations is an undesirable oil quality characteristic. In case of parental lines, the low proportion was recorded in BHAC-04026 (0.85\%), Entry no-23 (1.25\%) and BHAC-04038 (1.41\%) and stearic acid ranges in parental line $0.85-6.22 \%$, in selected lines for half diallel crosses it was lowest in Entry no-23 (1.25\%), BHAC-
04038(1) (1.78\%) and BHAC-P-S-2 (1.96\%) and it ranges 1.25-6.09. While in 28 derived crosses it recorded very low proportion from 1.96 to 5.17 percent. In case of hybrid low proportion was recorded in cross combination GP-01004x Entry-P-S-2 (1.96\%), GP-01009x Entry-23 (2.45\%) and GP-01004x GP-01005 (3.02\%). This result is in accordance with the report of Skoric et al. where they conclude stearic acid may vary $0.6-6.2 \%$ in sunflower, Praveen [4] recorded range of stearic acid $0.09-5.27 \%$ and stearic acid 4-6 per cent [5].

\section{Arachidic Acid}

Arachidic acid is also a saturated fatty acid which found $<1 \%$ in sunflower. High concentration of this fatty acid is undesirable for human health. FAO/WHO [6] reported arachidic acid is responsible for cardiovascular disease (DVD), coronary heart disease. In this study arachidic acid range in parental line was $0.13-0.53 \%$. The lowest proportion of arachidic acid found in the inbreds GP04019 (0.13\%), Entry no-22 (0.15\%) and Entry no-20 (0.20\%). In selected parent those were used in crossing programme in half diallel fashion ranges $0.20-0.46 \%$. In derived $28 \mathrm{~F} 1$ arachidic acid ranges from $0.20-0.39 \%$. The lowest proportion of arachidic acid in derived F1 was found in the cross combination GP-01004x GP01005 (0.1959\%), GP-01009x Entry-23 (0.2093\%), GP-01004x Entry-23 (0.2135\%) and GP-01004x Entry P-S-2 (0.2178\%).

\section{Unsaturated Fatty Acid (UFA)}

The sunflower oil had more than $90 \%$ of the unsaturated fatty acids and variation was noted among parents as well as hybrids 
regarding un-saturated fatty acid profile. Among unsaturated fatty acids, the linoleic and oleic is dominant in classical sunflower. There is an important genetic variation regarding the fatty acid composition of the sunflower oil. [7,8]. In this study UFA varied 47.67-91.68 percent which found in the inbred line GP04038(2) and BHAC-04038(1) respectively. The inbred lines BHAC-04028 (91.0535\%), Entry no.P-S-2(1) (90.4485\%), BHACSH-037 (90.3701\%) and Sun-W-103 (90.1689) contain more than $90 \%$ unsaturated fatty acid. Other inbreed contain $80-90 \%$ unsaturated fatty acid except only one inbreed GP-04038(2) which contain 47.66\% UFA. Kostik and Bauer; Skoric et al. [9] reported sunflower oil contain the highest percentage of long chain mono and polyunsaturated fatty acids than other vegetavles oil sources. According to FAO/WHO [6] UFA especially Poly Unsaturated Fatty Acid (PUFAs) is essential on human health in the prevention of particularly, cardiovascular disease (DVD), coronary heart disease.

\section{Oleic Acid}

High proportion of the essential fatty acid (18:2) is considered to reduce blood cholesterol and hence sunflower has a special significance [10]. It has greater oxidative stability and is useful as frying oil in the preparation of snack foods. High oleic acid also serves as a source of oleic acid useful in preparation of cosmetics and pharmaceuticals. The oleic acid proportion in sunflower genotypes varies from 30 to 90 percent and generally the genotypes have been classified into three classes viz., low oleic (10-29\%), mid oleic (30-59\%) and high oleic (60-90\%) (Lacombe and Bervillé, 2001, Pecureanu-Joita 2005). In present study the range of oleic acid varied greatly from 4.49 percent to 44.77 percent. Among 31 inbred majority of inbred viz. BHAC-04032, BHAC-P-S-2, BHAC-04038(1), BHAC-04026, BHAC-04016, Entry no-21, GP-01002, GP-01005, GP01009, GP-04011, GP-04012, GP-04015, GP-04016, GP-04017, GP04018, GP-04023, GP-04024, GP-04026, GP-04028, GP-04030 and GP-04038(2) are in low oleic group and 10 inbred viz. GP-04019, GP01004, Entry no.P-S-2(1), Entry no-23, Entry no-22, Entry no-20, BHAC-04028 and Sun-W-103 exhibits mid oleic content.

Among selected parent oleic acid ranges from 18.11-40.16\% (Table 3). Among derived crosses, four crosses were found to be higher oleic acid content viz., GP-01009x Entry P-S-2 (58.57\%), BHAC-04038(1) x GP-01005 (56.85\%), Entry no.-20 x GP-01009 (53.36\%) and BHAC-04038(1) x GP-01009 (52.05\%) compared to their respective parent. Whereas involving parents GP-01009, Entry P-S-2, BHAC-04038(1), GP-01005 and Entry no.-20 of these crosses contain 25.15\%, 38.90\%, 27.67\%, 18.11\% and $18.71 \%$ oleic acid (Table 4) respectively. Onemli (2012), Praveen (2015) explain significant variation for oleic acid while investigating fatty acid profile in sunflower oil. Kostik and Bauer [9] reported oleic acid ranges 27-36 percent, Rosa (2014) found oleic acid range 20$25 \%$, Praveen [4] observed oleic acid $24.63-84.97 \%$ in their study.

Table 4: Fatty acid profile of F1 generation of selected eight parents developed through half diallel fashion.

\begin{tabular}{|c|c|c|c|c|c|c|c|}
\hline \multirow{3}{*}{$\begin{array}{c}\text { Inbreds } \\
\text { Entry }\end{array}$} & \multicolumn{7}{|c|}{ Percentage of Fatty Acids } \\
\hline & \multicolumn{3}{|c|}{ Saturated Fatty Acid (SFA) } & \multicolumn{4}{|c|}{ Unsaturated Fatty Acid (USFA) } \\
\hline & $\begin{array}{c}\text { Palmitic } \\
\text { Acid (C16:0) }\end{array}$ & $\begin{array}{l}\text { Stearic Acid } \\
\text { (C18:0) }\end{array}$ & $\begin{array}{l}\text { Arachidc Acid } \\
\text { (C20:0) }\end{array}$ & $\begin{array}{l}\text { Oleic Acid } \\
\text { (C18:1) }\end{array}$ & $\begin{array}{c}\text { Linoleic } \\
\text { Acid (C18:2) }\end{array}$ & $\begin{array}{c}\text { Linolenic } \\
\text { Acid (C18:3) }\end{array}$ & $\begin{array}{c}\text { Cis-ecosenoic } \\
(20: 1)\end{array}$ \\
\hline Entry no.-20 x BHAC-04038(1) & 6.4547 & 3.367 & 0.2773 & 29.977 & 58.9471 & 0.0757 & 0.1525 \\
\hline Entry no.-20x GP-01004 & 4.6996 & 3.813 & 0.316 & 47.9099 & 42.2706 & 0.0772 & 0.1536 \\
\hline Entry no.-20x GP-01005 & 6.0676 & 3.6139 & 0.2955 & 34.0137 & 54.9263 & 0.0922 & 0.1400 \\
\hline Entry no.-20x GP-01009 & 5.4604 & 3.1893 & 0.2734 & 53.3699 & 36.5458 & 0.0997 & 0.2205 \\
\hline Entry no.-20x GP-04026 & 6.3626 & 4.1047 & 0.293 & 37.5991 & 50.36 & 0.0837 & 0.1244 \\
\hline Entry no.-20x Entry-23 & 6.5916 & 4.2828 & 0.2288 & 23.9345 & 64.0861 & 0.1079 & 0.1090 \\
\hline Entry no.-20x Entry P-S-2 & 5.7898 & 4.6149 & 0.2443 & 34.5122 & 53.9819 & 0.0755 & 0.0889 \\
\hline BHAC-04038(1)x GP-01004 & 5.8682 & 3.7117 & 0.2749 & 35.1755 & 53.9188 & 0.0978 & 0.1427 \\
\hline BHAC-04038(1)x GP-01005 & 4.581 & 3.089 & 0.2744 & 56.8591 & 34.2852 & 0.0010 & 0.1656 \\
\hline BHAC-04038(1)x GP-01009 & 5.0218 & 5.1661 & 0.3908 & 52.0538 & 35.5439 & 0.1535 & 0.2027 \\
\hline BHAC-04038(1)x GP-04026 & 5.7854 & 3.7338 & 0.245 & 34.8341 & 54.6678 & 0.0434 & 0.0949 \\
\hline BHAC-04038(1)x Entry-23 & 5.586 & 3.0349 & 0.2388 & 24.4293 & 65.8399 & 0.0878 & 0.0946 \\
\hline BHAC-04038(1)x Entry P-S-2 & 5.8882 & 4.1271 & 0.232 & 38.7072 & 50.1929 & 0.0831 & 0.2320 \\
\hline GP-01004x GP-01005 & 6.3964 & 3.023 & 0.1959 & 15.845 & 73.7622 & 0.0866 & 0.1344 \\
\hline GP-01004x GP-01009 & 6.5095 & 5.0203 & 0.3753 & 25.1674 & 61.8882 & 0.0642 & 0.0985 \\
\hline GP-01004x GP-04026 & 5.4795 & 3.4541 & 0.2618 & 35.363 & 54.2463 & 0.0751 & 0.1628 \\
\hline GP-01004x Entry-23 & 6.8521 & 3.67 & 0.2135 & 20.73 & 67.8152 & 0.1148 & 0.1102 \\
\hline GP-01004x Entry P-S-2 & 5.8558 & 1.9563 & 0.2178 & 39.7321 & 51.3652 & 0.0917 & 0.1497 \\
\hline GP-01005x GP-01009 & 5.4077 & 3.0269 & 0.2896 & 32.8015 & 57.3424 & 0.0709 & 0.0010 \\
\hline GP- $01005 x$ GP-04026 & 6.293 & 3.9712 & 0.2833 & 24.0317 & 64.4185 & 0.079 & 0.1218 \\
\hline
\end{tabular}




\begin{tabular}{|c|c|c|c|c|c|c|c|}
\hline GP-01005x Entry-23 & 6.6211 & 3.7264 & 0.2925 & 25.9668 & 62.4511 & 0.0535 & 0.0805 \\
\hline GP-01005x Entry P-S-2 & 6.2656 & 4.623 & 0.3019 & 26.1454 & 61.0895 & 0.2033 & 0.2124 \\
\hline GP-01009x GP-04026 & 5.8085 & 3.3758 & 0.2916 & 41.6559 & 47.8395 & 0.1797 & 0.2491 \\
\hline GP-01009x Entry-23 & 5.0227 & 2.4532 & 0.2093 & 35.9909 & 55.4332 & 0.07 & 0.1583 \\
\hline GP-01009x Entry P-S-2 & 4.9687 & 3.3028 & 0.3448 & 58.5712 & 31.3926 & 0.1067 & 0.1673 \\
\hline GP-04026x Entry-23 & 6.882 & 3.6665 & 0.3022 & 40.1891 & 47.7305 & 0.1157 & 0.135 \\
\hline GP-04026x Entry P-S-2 & 6.0189 & 4.3524 & 0.2563 & 32.776 & 55.4772 & 0.1489 & 0.1313 \\
\hline Entry-23x Entry P-S-2 & 5.7706 & 4.4571 & 0.2777 & 26.5717 & 61.9086 & 0.1987 & 0.1626 \\
\hline Mean & 5.87 & 3.71 & 0.27 & 35.18 & 53.92 & 0.10 & 0.14 \\
\hline Standard Deviation & 0.63 & 0.73 & 0.05 & 10.94 & 10.57 & 0.04 & 0.05 \\
\hline CV & 10.71 & 19.66 & 16.92 & 31.10 & 19.60 & 45.93 & 36.40 \\
\hline Minimum & 4.58 & 1.96 & 0.20 & 15.85 & 31.39 & 0.01 & 0.01 \\
\hline Maximum & 6.88 & 5.17 & 0.39 & 58.57 & 73.76 & 0.20 & 0.25 \\
\hline
\end{tabular}

\section{Linoleic Acid}

In traditional sunflower oil, the linoleic acid content will be generally very high (60-70\%). Linoleic acid and its derivative fatty acids are essential fatty acids and not synthesized by human being and hence, must be obtained from dietary sources. High level of linoleic acids in the oil reduces the blood cholesterol level and plays an important role in preventing atherosclerosis [11]. Thus, edible oil with high linoleic acid content is premium oil. According to results of this study Entry no-20 had highest linoleic acid (69.89\%) among the studied inbreds which can be used for genetic analysis and breeding programs. Among 31 inbreds line linoleic acid ranges from 18.37-69.89. Others inbred GP-01005 (67.81\%), GP-04017 $(67.40 \%)$ and GP-04012 (67.34\%) also contain high percent of lenoleic acid which can be utilized in quality breeding to develop healthy sunflower oil. In crossing generation of selected line linoleic acid ranges 31.39-73.76\%. Among derived crosses, the highest proportion of linoleic acid recorded in combination GP-01004x GP01005 (73.76\%), GP-01004x Entry-23 (67.81\%), BHAC-04038(1) x Entry-23 (65.84\%) and GP-01005x GP-04026 (64.42\%) compare to their respective parents. Whereas involving parents GP-04026, BHAC-04038(1), Entry-23, GP-01005 and GP-01004 contain $66.22 \%, 63.87 \%, 55.99 \%, 67.82 \%$ and $46.72 \%$ respectively (Tables 2 \& 3). Kostik and Bauer [9] reported 52-67 percent, Rosa et al. [12] 62-69\%, Praveen (2015) 24.63-84.97\%, Skoric (2015) 2.687.1\% linoleic acid in their study. Onemli [3], Praveen [4] conclude linolenic acid varied significantly in sunflower germplasm.

\section{Linolenic Acid}

It is worthy to note that linolenic acid is also an essential fatty acid; however, its presence in the oil may causes rancidity and offflavor. In traditional sunflower oil, the linolenic acid content will be generally $<1$ percent. As like as linoleic acid, linolenic acid and its derivative fatty acids are essential fatty acids and not synthesized by human being and hence, must be obtained from dietary sources. In this study the inbred Entry no-22 and GP-04019 had highest linolenic acid $0.4276 \%$ and $0.4234 \%$ among the studied lines which can be used for genetic analysis and breeding programs. Others inbred GP-04017 (0.3181\%), GP-01009 (0.2323), GP04026 (0.1585\%), GP-04018 (0.1576\%), GP-04028 (0.1480\%) and GP-04024 (0.1447\%) also contain high percent of lenolenic acid which can be utilized in quality breeding to develop healthy sunflower oil. In selected lines it ranges 0.06-0.23. In F1 obtain from selected parents it ranges $0.01-0.02 \%$. Among derived crosses, the highest proportion of linolenic acid recorded in combination GP01005x Entry P-S-2 (0.20\%), Entry-23x Entry P-S-2 (0.20\%), GP01009x GP-04026 (0.18\%) and GP-04026x Entry P-S-2 (0.15\%) respectively. Whereas involving parents GP-04026, GP-01009, Entry-23, Entry P-S-2 and GP-01005 contain 0.0591\%, 0.0668\%, $0.0987 \%, 0.2323 \%$ and $0.1585 \%$ respectively (Table 3 ). Onemli [3], Praveen [4] reported significant variation for linolenic (C18:3) acid in their study.

\section{Cis-ecosenoic Acid}

Cis-ecosenoic acid is also unsaturated fatty acid which found in sunflower genotype $<1$ percent. Onemli [3], Praveen [4] reported significant variation for cis-ecosenoic acids (C20:1) in their study. In parental line in this study its range is $0.0004-1.00$ percent. The genotype entry no-21(1.00\%) and Entry no-22(1.00\%) contain the highest percentages of cis-ecosenoic acid in parental inbred. Selected germplasm it ranges 0.001-0.79 percent. The selected inbred GP-04026 (0.79\%) contain the highest proportion of cisecosenoic acid. In derived crosses it ranges $0.01-0.25 \%$. The highest proportion of cis-ecosenoic acid found in the cross combination GP-01009x GP-04026 (0.25\%), BHAC-04038(1)x Entry P-S-2 (0.2320\%), Entry no.-20x GP-01009 (0.2205\%) and GP-01005x Entry P-S-2 (0.2124\%) respectively [13-21].

\section{References}

1. Gossal SS, Vasiljevic L, Brar DS (1988) Plant biotechnology and sunflower improvement. In Proceedings of $12^{\text {th }}$ International Sunflower Conference. Novisad Yugoslavia, p. 25-29.

2. Alvarez Ortega R, Cantisán S, Martínez Force E, Garcés R (1997) Characterization of polar and nonpolar seed lipid classes from highly saturated fatty acid sunflower mutants. Lipids 32(8): 833-837. 
3. Onemli F (2012) Impact of climate changes and correlations on oil fatty acids in sunflower. Pak J Agri Sci 49(4): 455-458.

4. Praveen HG (2015) Genetic variability for oil content and fatty acid composition in germplasm accessions of sunflower (Helianthus annuus L.) university of agricultural sciences gkvk, bengaluru.

5. Anonymous (2015-2016) Annual Report: Sunflower. Directorate of Oilseed Research, Hyderabad 10: 15-16.

6. World Health Organization (2015) Evaluation of certain food additives: seventy-ninth report of the Joint FAO/WHO Expert Committee on Food Additives, pp. 990.

7. Cummins DG, Marion JE, Craigmiles JP, Burns RE (1967) Oil content, fatty acid composition and other agronomic characteristics of sunflower introductions. J Am Oil Chem Soc 44(10): 581-582.

8. Simpson BW, George DL (1985) Potential for selection of fatty acids on a single seed basis in sunflower (Helianthus annuus L.). Proc $11^{\text {th }}$ Intern Sunflower Conf, Mar del Plata, Argentina (2): 791-796.

9. Kostik V, Memeti S, Bauer B (2013) Fatty acid composition of edible oils and fats. Journal of Hygienic Engineering and Design 4:112-116.

10. Flagella Z, Rotunno T, Tarantino E, Di Caterina R, De Caro A (2012) Changes in seed yield and oil fatty acid composition of high oleic sunflower (Helianthus annuus L.) hybrids in relation to the sowing date and the water regime. European J Agron 17: 221-230.

11. Miller JF, Zimmerman DC, Vick BA (1987) Genetic control of high oleic acid content in sunflower oil 1. Crop Science 27(5): 923-926.

12. Rosa PM, Antoniassi R, Freitas SC, Bizzo HR, Zanotto DL, et al. (2014) Chemical composition of brazilian sunflower varieties. Helia 32(50): 145-156.

ISSN: 2574-1241

DOI: 10.26717/BJSTR.2019.16.002851

Mahmmmed Harun. Biomed J Sci \& Tech Res

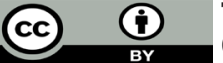

This work is licensed under Creative Commons Attribution 4.0 License

Submission Link: https://biomedres.us/submit-manuscript.php
13. Izquierdo NG, Aguirrezábal LA (2008) Genetic variability in the response of fatty acid composition to minimum night temperature during grain filling in sunflower. Field Crops Research 106(2): 116-125.

14. Lacombe S, Souyris I, Bervillé AJ (2009) An insertion of oleate desaturase homologous sequence silences via siRNA the functional gene leading to high oleic acid content in sunflower seed oil. Molecular Mol Genet Genomics 281: 43-54.

15. Marmesat, Susana, Leonardo Velasco, Maria Victoria Ruiz-Méndez, José María Fernández Martínez, Carmen Dobarganes (2008) Thermostability of genetically modified sunflower oils differing in fatty acid and tocopherol compositions. Eur J Lipid Sci Technol 110(8): 776-782.

16. Nazir S, Bashir E, Bantel R (1994) Crop production. National Book Foundation, Islamabad, pp. 348.

17. Pecureanu-Joita M, Stanciu D, Petcu E, Raranciuc S, Sorega I (2005) Sunflower genotypes with high oleic acid content. Romanian Agric Res 22: $23-26$.

18. Satyabrata M, Hedge MR, Chattopodhay SB (1988) Hand book of annual oilseed crops. Oxford \& IBH, pp. 325.

19. Seiler GJ, Gulya TJ, Fredrick-Marek L (2012) Collection and evaluation of wild perennial Helianthus pumilus achenes for oil concentration and fatty acid composition. In $18^{\text {th }}$ International Sunflower Conference program and abstracts. Argentina, pp. 175.

20. Shahidi F (2005) Bailey' s industrial oil and fat products, (Volume 4), edible oil and fat products: Products and applications. Wiley, pp. 600.

21. Yadav P, Tilak IS, Kisan B, Goud IS, Naika MB, et al. (2018) Biochemical and molecular characterization of parents and its crosses for high oleic acid content in sunflower (Helianthus annuus L.). Int J Curr Microbiol App Sci 7(4): 2000-2020.

$\begin{array}{ll}\text { BIOMEDICAL } & \text { Assets of Publishing with us } \\ \text { RESEARCHES } & \text { - Global archiving of articles } \\ & \text { - Immediate, unrestricted online access } \\ & \text { - Rigorous Peer Review Process } \\ \end{array}$

